\title{
Muon trigger counters for a hadron collider experiment
}

\author{
J.W. Chapman ${ }^{\text {a }, ~ E . ~ D o d d ~}{ }^{\text {a }}$, S. Hong ${ }^{\text {a }}$, J. Mann ${ }^{\text {a }}$, R. Thun ${ }^{\mathrm{a}, *}$, C. Weaverdyck ${ }^{\mathrm{a}}$, K. Yale ${ }^{\mathrm{a}}$, \\ V. Kubarovsky ${ }^{\text {b }, ~ V . ~ R y k a l i n ~}{ }^{\mathrm{b}}$ \\ "Unuversity of Michigan, Ann Arbor, MI 48109, USA \\ ${ }^{b}$ Institute for High Energy Phystcs, Protvino, Moscow Region, 142284, Russian Federation
}

(Recelved 4 January 1994)

We describe the design and performance of prototype trigger counters for the SDC muon system. The $1.0 \times 48.7 \times 178.7 \mathrm{~cm}$ scintillation counters feature compact light guides, use standard 2 in. phototubes, and provide good photoelectron yields $(>25)$ and time resolution $(<1 \mathrm{~ns})$.

\section{Introduction}

We report in this paper results from an effort to develop a muon trigger counter system for SDC, a general-purpose detector proposed for the Superconducting Super Collider (SSC). Although SSC has been terminated, these results may find application in other collider experiments. As indicated in Fig. 1, the SDC detector [1] consists of three major subsystems: an inner tracker immersed in a solenoidal magnetic field, a calorimeter, and an exterior muon detector. The muon system provides a muon trigger with a variable momentum threshold and is capable of identifying the beam crossing that generates the trigger.

In the SSC design, beam bunches cross every 16 ns at the interaction point. Unambiguous identification of the bunch crossing requires a muon trigger time resolution of at least $2 \mathrm{~ns}$ (rms). This timing requirement, coupled with the necessity to cover an area of more than $2000 \mathrm{~m}^{2}$ with a reliable, well-proven detector technology, led us to choose plastic scintillators for the timing function. Initially, we studied a number of scintillator configurations, including some with wave-shifting readout fibers, but eventually settled on a geometry consisting of narrow, long counters read out at both ends through fairly standard light guides that are folded back across the counter. Instrumenting each counter with two photomultiplier tubes (PMTs) allows the application of mean-timing techniques that automatically remove the time dispersion introduced in long counters by the finite light propagation speed of about $16 \mathrm{~cm} / \mathrm{ns}$.

\footnotetext{
* Corresponding author.
}

Requiring coincident signals from each counter also eliminates spurious triggers from PMT noise.

Originally, we proposed two layers of scintillators around SDC to reduce random backgrounds from the high levels of low-energy photons and neutrons expected at SSC. Detailed estimates of these backgrounds coupled with the necessity to minimize cost, led us to reduce this to one layer in the large-angle (barrel) region of SDC. As indicated in Fig. 1, the muon counter layout of SDC consists therefore of a single layer of scintillators located outside the toroidally magnetized iron barrel, and two layers behind the forward iron toroids, a region of more intense backgrounds.

The basic SDC muon trigger concept, illustrated in Fig. 2, is based on a coincidence of signals from muon drift tubes and trigger counters. Momentum thresholds are imposed by placing an upper limit on the deflection angle of charged particles emerging from the iron toroids. This is accomplished by limiting the allowable differences in drift times measured in pairs of proportional tubes aligned projectively with respect to the interaction point. Scintillation counters that overlap the drift tubes provide the time information that allows the drift-tube signals to be correlated to a particular beam crossing. Because the drift times can be as long as $900 \mathrm{~ns}$ in the SDC design, the muon trigger electronics stretches the drift-tube signals and delays the scintillator output by an amount equal to the maximum drift time to ensure coincidence.

In the following sections we describe the design and performance of prototypes of the muon counters in the single barrel layer of SDC. We then address some issues related to calibrations and to the long-term use of these counters in a high-luminosity environment. A facility that would have tested all SDC muon counters 


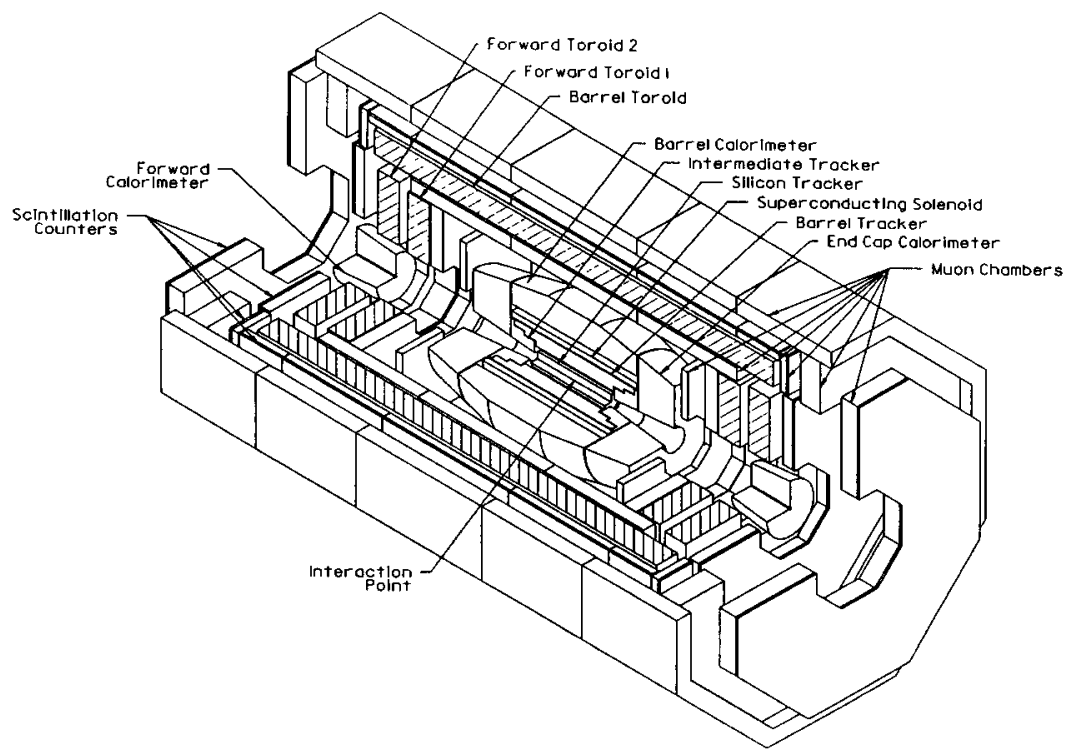

Fig. 1. Proposed SDC detector.

was constructed as part of the overall research and development effort and this is also described below.

\section{Counter design and system layout}

The basic layout of the SDC muon counters is sketched in Fig. 3 for one of the octants of the barrel

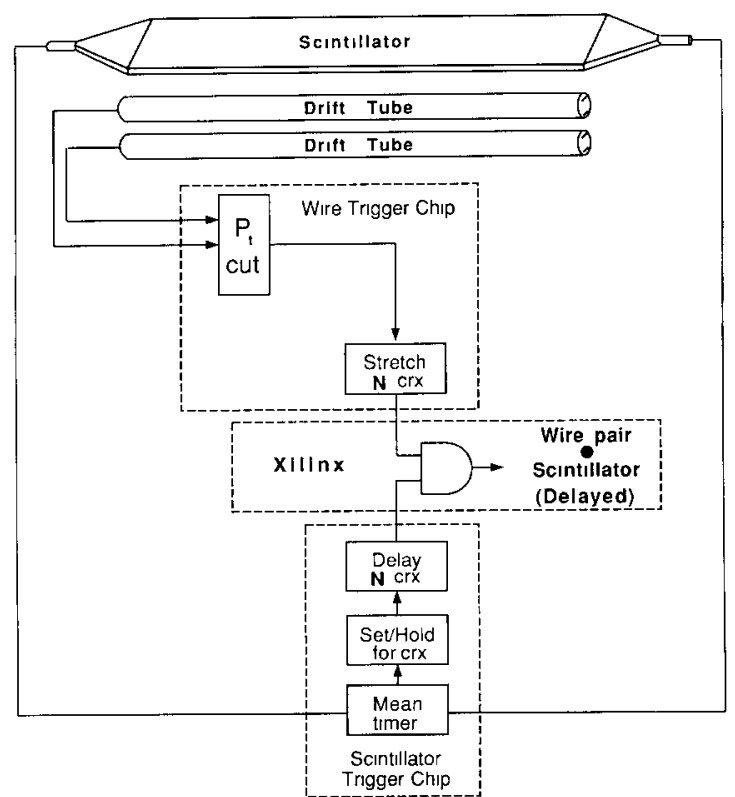

Fig. 2. SDC muon trigger scheme ("crx" stands for SSC bunch crossing period). toroid. The counters are located on the outer surface of drift-tube module BW2 which, in turn, is attached directly to the exterior of the iron toroid. The width of each octant is spanned by four identical counters whose configuration and dimensions are shown in Figs. 4 and 5 . To maximize coverage, the four counters have minimal gaps (about $3 \mathrm{~mm}$ ) between their narrow sides, and adjacent rows of four counters are slightly overlapped when viewed from the interaction point. This overlapping geometry is illustrated in Figs. 6 and 7. Each scintillation counter is held by a rigid aluminum frame that is mounted via retractable pins to aluminum rails attached directly to the BW2 drift-tube modules. The aluminum frame supports the weight of the light guides, magnetic shields, photomultipliers (PMTs) and bases, thereby minimizing mechanical stresses on the scintilla-

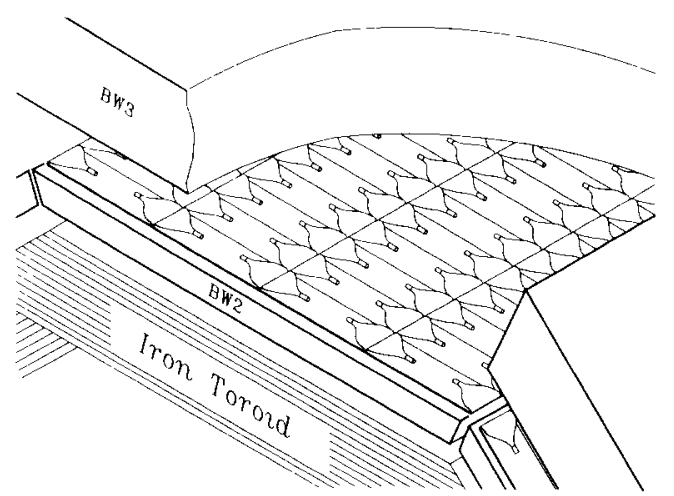

Fig. 3. Layout of SDC barrel muon counters. BW2 and BW3 are muon tracking modules. 


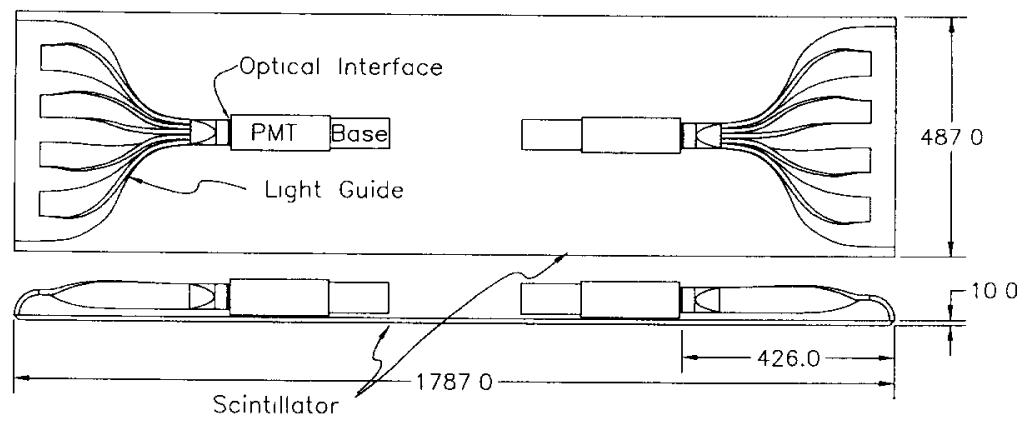

Fig. 4. Configuration of scintillator and light guides of SDC barrel muon counter with dimensions in $\mathrm{mm}$.

tor itself. (The total mass per counter, including magnetic shields, is approximately $25 \mathrm{~kg}$.) This design feature reduces the risk of mechanically-induced scintillator crazing.

The counter geometry was dictated by a number of considerations. The light guides are folded back across the top of the scintillators so that all PMTs and bases are accessible without moving any counter. Easy access is a critical requirement since the radial separation of $1.0 \mathrm{~m}$ between drift-tube modules BW2 and BW3 (see Fig. 3) represents a very tight space for mounting and servicing the muon trigger counters. The design also maximizes scintillator coverage at the octant boundaries since the light guides do not intrude into the space between octants.

The total counter length of just under $1.8 \mathrm{~m}$ was the maximum that would allow individual counters to be easily extracted from SDC without dismantling major portions of the detector. Moreover, this length limit was also consistent with keeping light attenuation and light transit times within reasonable bounds. The

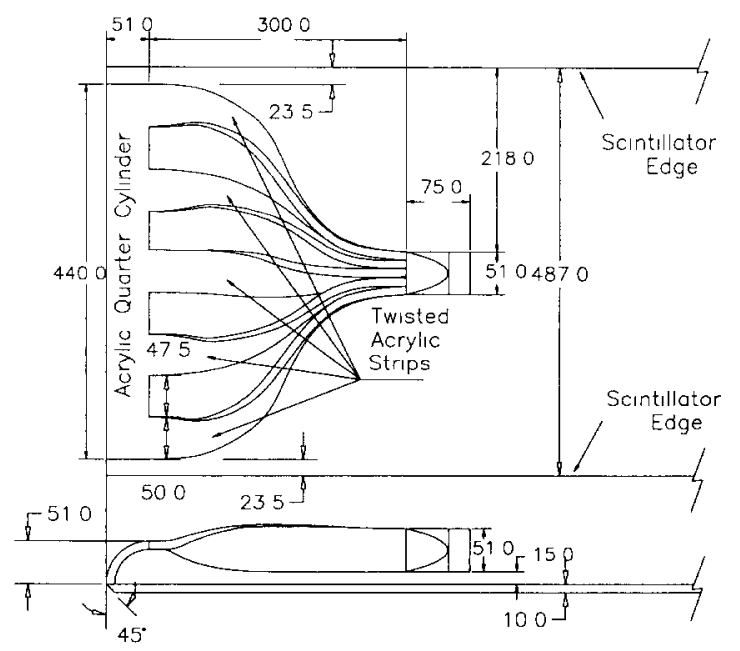

Fig. 5. Detailed view of light guide region of SDC barrel muon counter. counter thickness of $10 \mathrm{~mm}$ and width of just under 0.5 $\mathrm{m}$ were chosen to obtain a reasonable number of photoelectrons $(>25$ ) for muons striking any portion of the counter when using widely available 2 in. diameter PMTs. The scintillator thickness not only affects cost and weight but also the relative light yield from muons and low-energy background neutrons and photons. The choice of counter and PMT dimensions represents a complex problem of optimizing cost vs performance. The prototype counters described here represent a preliminary, intuitive solution since final cost estimates were not completed before SSC was terminated.

The task of reversing the path of scintillation photons is accomplished in two steps. The first consists of internal reflection at the scintillator ends which are cut at $45^{\circ}$ angles as shown in Fig. 5 [2]. A quarter section of cylindrical tubing then continues the internal reflection of photons to a light guide consisting of five twisted strips that join at the PMT. The tubing and strips are polished acrylic plastic. The scintillator is glued to the light guide with a polyurethane adhesive [3] that forms a strong bond with moderate flexibility. The light-guide components are glued to each other with acrylic cement [4].

We compared the light-transmission properties of this counter design, with its "180-degree" light guide, to those obtained with a standard configuration in which the light guide was glued directly to a straight scintillator edge, leaving off the intermediate quartercylindrical section. Measurements of average photoelectron yields from cosmic rays were made with the "zero-degree" and "180-degree" light guide using the same scintillator, PMT, base, and operating voltage. The "180-degree" light guide yielded $0.65 \pm 0.06$ as many photoelectrons as the "zero-degree" guide. We decided to accept this reduction in yield to obtain a compact, accessible counter design.

We tested a number of methods of coupling the light guide to the PMT that would allow easy removal of the PMT in case of failure. The results are summarized in Fig. 8 which shows the response of a small 


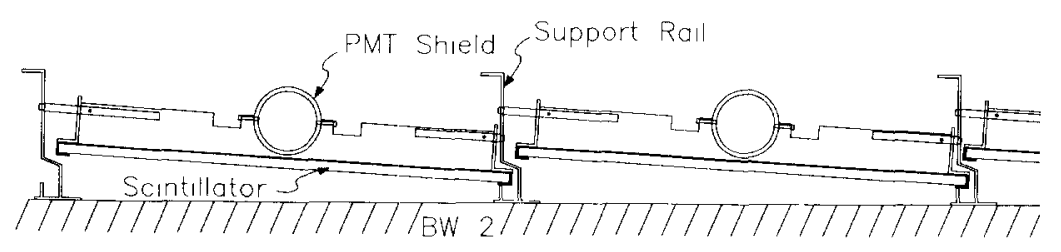

Fig. 6. End view of layout of SDC barrel muon counters showing overlapping geometry.

scintillation counter to cosmic rays as a function of coupling scheme. Tested configurations included simple physical contact (the "air gap" data point in Fig. 8), coupling with only optical grease [5] (the "no disk" data point), and optical interface disks with and without the use of optical grease. We tested commercially available $4.6 \mathrm{~mm}$ thick disks [6] (the "Bicron" data point) as well as $6.4 \mathrm{~mm}$ thick disks produced in our laboratory using the two-component silicone rubber RTV615 [7] (the data points for mix ratios of 10, 17. 25, and 30). The mechanical stiffness of the optical disks is a function of mix ratio of the two components, with low

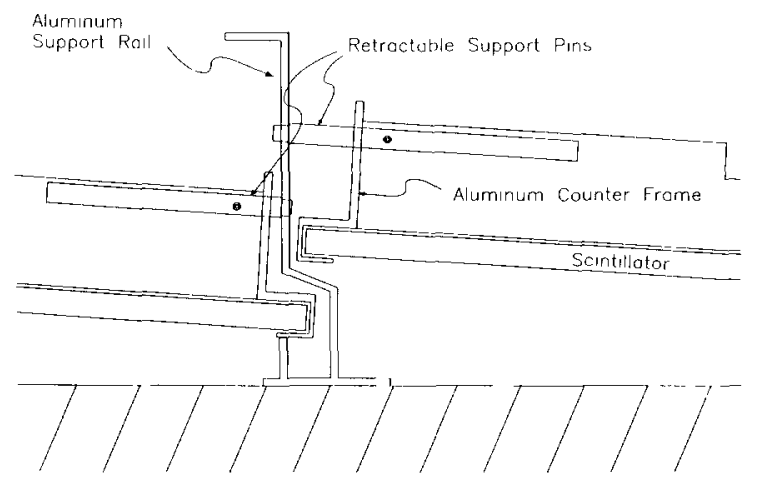

Fig. 7. Detailed view of mountıng scheme for SDC barrel muon counters.

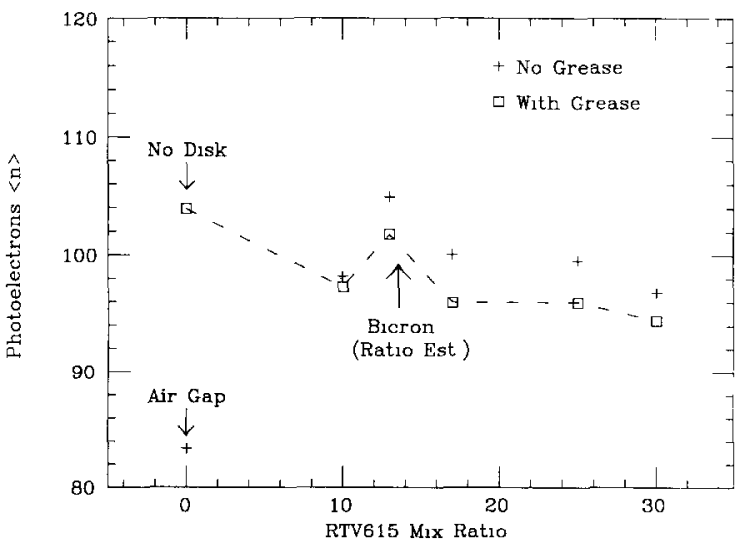

Fig. 8. Relative efficiency of various optical interfaces between light guide and PMT. mix ratios giving the hardest disks. We estimated that the Bicron disks have a stiffness equivalent to an RTV615 mix ratio of 13 (see Fig. 8). We concluded that the use of optical interface disks without grcase represents the best compromise with regard to efficiency, mechanical properties, and serviceability [8]. The harder disks are preferable because of their robustness and well-defined resistance to compression.

Magnetic fringe fields from the coils of the SDC barrel toroid are estimated to produce fields of up to about $80 \mathrm{G}$ at the location of the muon counter PMTs. Preliminary computations indicated that magnetic shiclds consisting of nested cylinders of soft iron and a high-permeability alloy would reduce such fields to below I G at the PMT. Planned tests of optimal shiclding configurations were cancelled as a consequence of SSC termination.

\section{Mean timer}

We use a mean-timing technique to obtain signal arrival times that are independent of where muons strike the trigger counter. As shown in Fig. 9, signals from oppositc ends of a trigger counter (labeled "PL" and "PR" in Fig. 9) are fed into a digital mean timer (DMT) with opposing delay chains linked by a series of eight coincidence circuits. For a single hit sensed by both ends of the counter, the coincidence output time

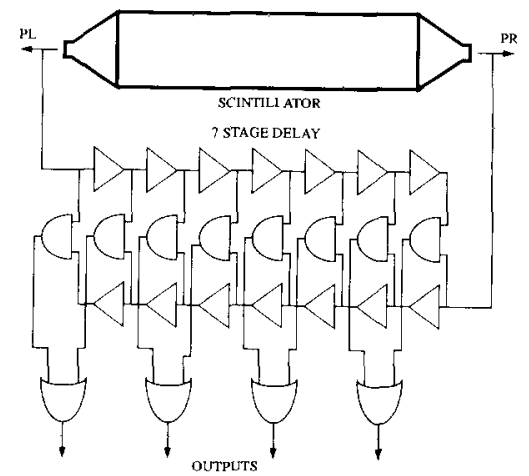

Fig. 9. Schematic of mean timer for SDC muon trigger counters. 
is independent of hit location. The position of the hit can be determined by checking which section of the coincidence chain gives the output signal. As indicated in Fig. 9, these position-sensitive signals are paired in order to divide the scintillator into four logical segments, approximately $49 \mathrm{~cm}$ wide and $45 \mathrm{~cm}$ long. To achieve accurate segmentation of the counter length, the delay per stage of the DMT is programmable over a 10-14 ns range for the combined seven delay steps.

For multiply-struck counters, unresolvable hits shift the mean-timer output by one half of the difference in light transit times between hits. For the SDC trigger counters, this represents a maximum shift of less than $6 \mathrm{~ns}$, well within the $16 \mathrm{~ns}$ bunch crossing period. The DMT is deadtimeless for PMT signals that produce distinct discriminator outputs. A VME trigger module has been constructed with the scintillator DMT and ASICs that process the drift tube signals. This trigger module will be the topic of another paper.

To measure the time resolution of the SDC prototype trigger counters, we designed and constructed several NIM DMT modules [9] from early versions of the DMT ASIC which lacked the position-locating outputs. These prototypes were fabricated by MOSIS and contain a 16-stage mean timer and digital delay buffers that are phase locked to a precise $2.0 \mathrm{~ns}$ propagation delay per stage. The unit contains a programmable window that enables coincidence circuits symmetrically around zero time difference between the PMT signals from the opposite ends. This window was set to accept PMT signals that could have come from a single track passing the scintillator. The DMT includes circuitry to shape the incoming pulses to approximately 5.0 ns width for the mean timer inputs. In the NIM module there are converters to translate to and from NIM levels and to widen the relatively narrow pulse from the mean timer for output in NIM levels on the front panel.

\section{Counter performance}

The photoelectron yield and time resolution of the counters were measured with cosmic rays. Several test facilities were employed and gave consistent results. One of these consisted of a pair of small $(15 \times 15 \mathrm{~cm})$, vertically separated scintillation counters that were used to define cosmic-ray impacts in selected regions of the counter to be tested. Signals from the test-counter PMT were then processed by a pulse-height analyzer (PHA). More elaborate cosmic-ray test stands that included tracking capabilities and a sophisticated data acquisition system (with ADCs and TDCs) were also used to measure the response of prototype counters. One such test stand is described in section 7.

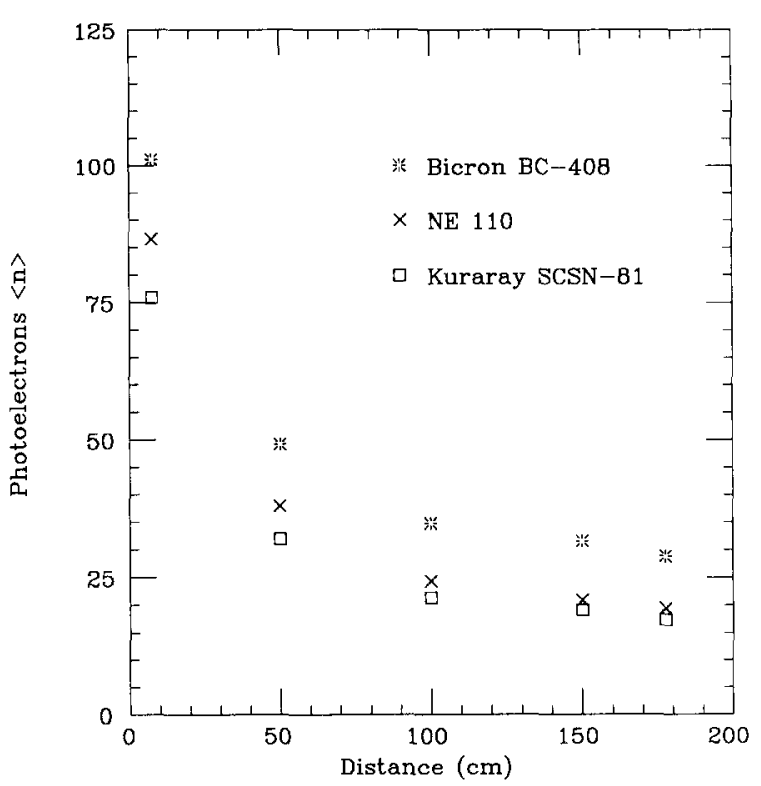

Fig. 10. Response to cosmic rays of several identically sized and instrumented scintillators as a function of distance from the light guide.

Photoelectron yields were measured by triggering the test counters with light-emitting diodes (LEDs) and using the width of the resulting Gaussian-shaped signals to calibrate the PHA or ADC scales. During the early stages of our scintillator research, we compared the relative light yields of a number of commonly used scintillators. Typical results are shown in Fig. 10 which displays the average photoelectron yield from cosmic rays as a function of distance from the end of identically sized scintillators $(1.0 \times 50 \times 185 \mathrm{~cm})$. The same light guide (a simple, $15 \mathrm{~cm}$ wide, tapered piece of acrylic), PMT (Hamamatsu R329), base, and operating voltage were used with each scintillator. For these measurements, the scintillator edge opposite the PMT was covered with black tape to avoid reflections. The initial, sharp decrease in light yield with distance arises partially from the mismatch of widths between the test light guide $(15 \mathrm{~cm})$ and counter $(50 \mathrm{~cm})$. Bicron BC-408 gave consistently higher light yields and longer attenuation lengths than either NE 110 or Kuraray SCSN-81, the latter two having approximately equal performance. The Bicron and NE scintillators are based on polyvinyltoluene (PVT) whereas Kuraray uses polystyrene (PS).

Muon counter prototypes built to the specifications and dimensions shown in Fig. 4 were constructed with Bicron BC-408 and Kuraray SCSN-81. (We also used NE 110 for larger counters $(1.0 \times 66 \times 250 \mathrm{~cm})$ of the same general design for use in the cosmic-ray test stand described in section 7.) The Kuraray prototype 


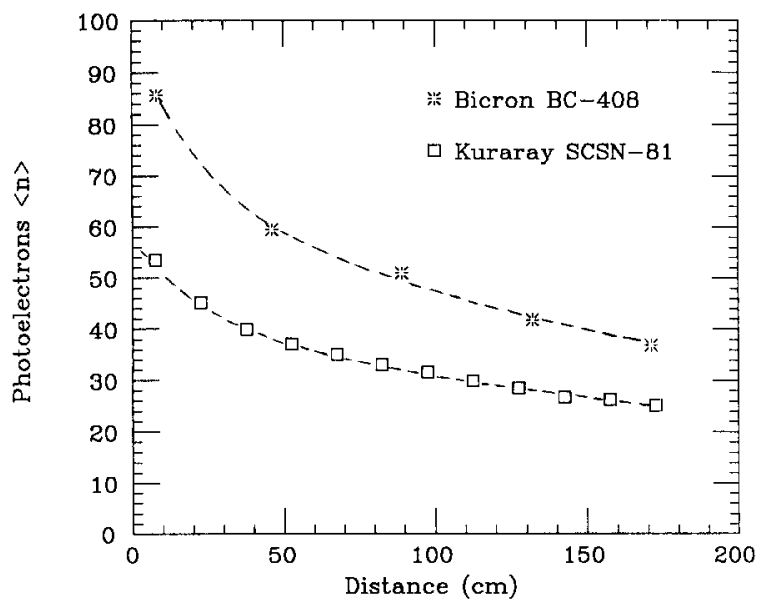

Fig. 11. Response to cosmic rays of two SDC prototype muon counters as a function of distance from the counter end.

was instrumented with Hamamatsu R329 PMTs and the Bicron counter with Burle 8575 PMTs, both 2 in. diameter, 12-stage photomultipliers. The measured photoelectron yield from cosmic rays is shown in Fig. 11 as a function of distance from the counter end. We also tested a number of prototype counters produced by the Institute for High Energy Physics (IHEP) at Protvino, Russia [10]. These polystyrene-based counters were instrumented with 2 in. diameter, 10-stage FEU-184 PMTs. Initial IHEP counters were extruded without further machining or polishing (except at the $45^{\circ}$ edges); later versions have all surfaces machined and polished. As shown in Fig. 12, the performance of the polished IHEP counters is roughly comparable to the Bicron and Kuraray counters, with somewhat greater light attenuation apparent in the IHEP scintillator.

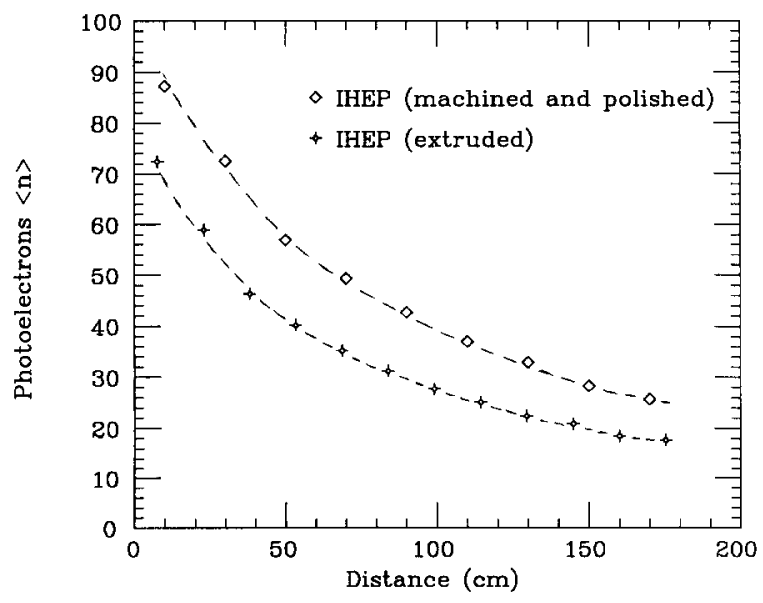

Fig. 12. Response to cosmic rays of SDC prototype counters using two types of IHEP scintillator.

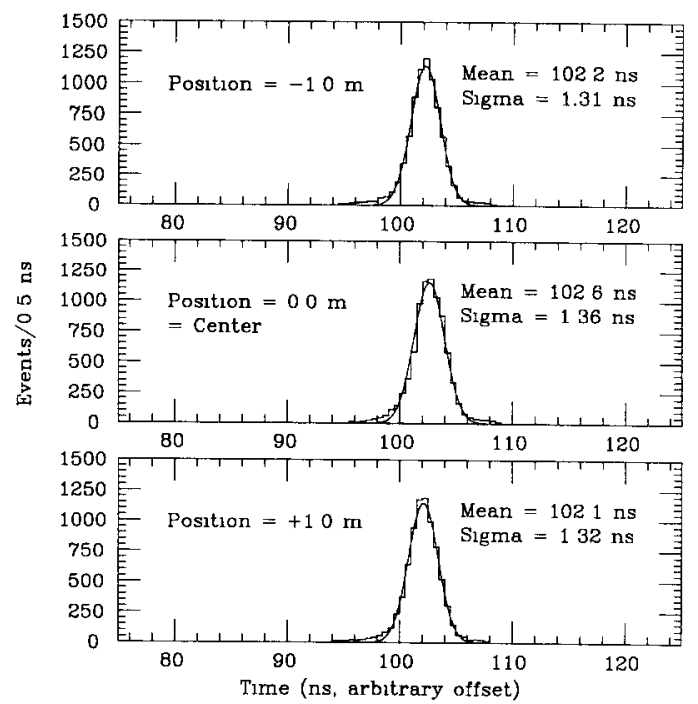

Fig. 13. Mean-timer output from three different locations on a $2.7 \mathrm{~m}$ long test counter. The time resolution includes the time jitter (1.07 $\mathrm{ns} \mathrm{rms})$ of the cosmic-ray telescope that defined incident tracks.

As described in section 3, trigger-counter coincidence signals are generated with a mean timer whose output time is independent of the location of the triggering particle. Fig. 13 shows the time distributions obtained by triggering a $2.7 \mathrm{~m}$ long scintillator with cosmic rays at three locations along a $2.0 \mathrm{~m}$ segment of the counter. The means of the time distributions differ by less than $0.5 \mathrm{~ns}$. The average resolution of $1.33 \mathrm{~ns}$ (rms) is dominated by the time resolution $(1.07 \mathrm{~ns})$ of the cosmic-ray telescope which provided the start signal for the time digitization. The intrinsic resolution of the counter itself is estimated to be $0.8 \mathrm{~ns}$ (rms). Measurements of the time response of the SDC muon trigger counter prototypes gave similar results.

\section{Scintillator crazing study}

Detectors for modern collider experiments require long construction periods and are expected to perform measurements for a decade or more. This raises serious questions about long-term stability which, for scintillation counters, is affected by aging and radiation damage. The barrel trigger counters are located in a region of low radiation levels, of order 10 rad or less per year at design luminosity, so that effects other than radiation damage may dominate detector degradation. Long-term tests of polystyrene-based scintillators [11] indicate light-yield losses of a few percent per year from normal aging processes in the scintillator material. A potentially more serious concern for any plastic 


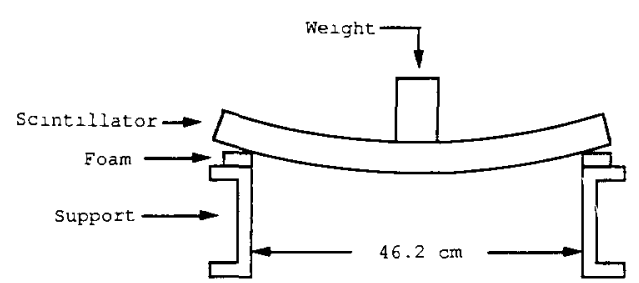

F1g. 14. Setup for tests of mechanically induced scintillator crazing.

scintillator is the possibility of significant light loss from crazing, a phenomenon in which scintillator surfaces develop a dense pattern of small cracks induced by chemical or mechanical stresses.

We tested the susceptibility to mechanically-induced crazing [12] with the setup shown in Fig. 14. Pieces of scintillator manufactured by Bicron, IHEP, Kuraray, and NE were placed on simple supports and then stressed with weights placed at the center of the scintillator. The dimensions of the scintillators used in this test are approximately $15 \times 57 \mathrm{~cm}$ with varying thicknesses as indicated in Figs. 15 and 16. The weights were chosen to provide the same fiber stress in all but one of the pieces, corresponding to about $10 \%$ of the yield strength of the Bicron scintillator. For example, the $8.85 \mathrm{~kg}$ mass placed on the $1.24 \mathrm{~cm}$ thick BC-408 gave a maximum fiber stress of about $2.6 \mathrm{MPa}$.

The stressed scintillators were inspected visually at regular intervals. Significant crazing was observed within a few days in all PVT-based scintillators (Bicron and NE) and was initially localized on the bottom surfaces, just below the weight and along the side edges. Over a period of several weeks, the crazing

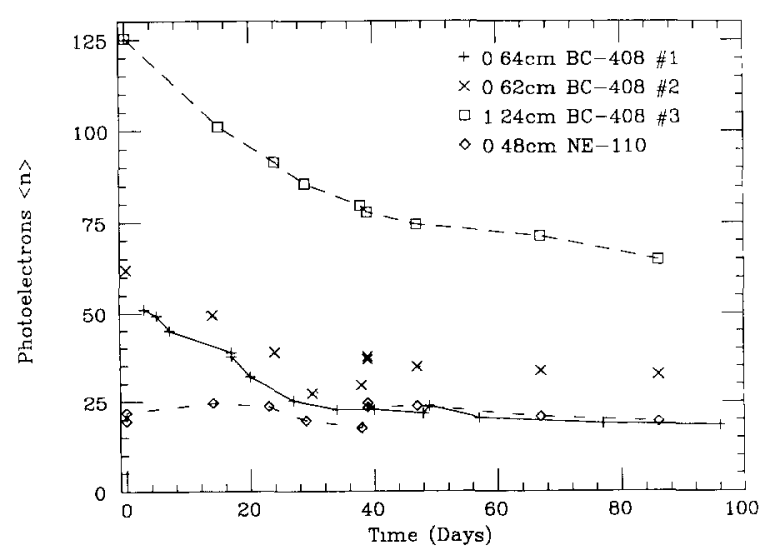

Fig. 15. Response of mechanically stressed PVT-based scintıllators of various indicated thicknesses as a function of time under stress. Some measurements just prior to the 40 -day mark may have been affected by an inadvertent light leak.

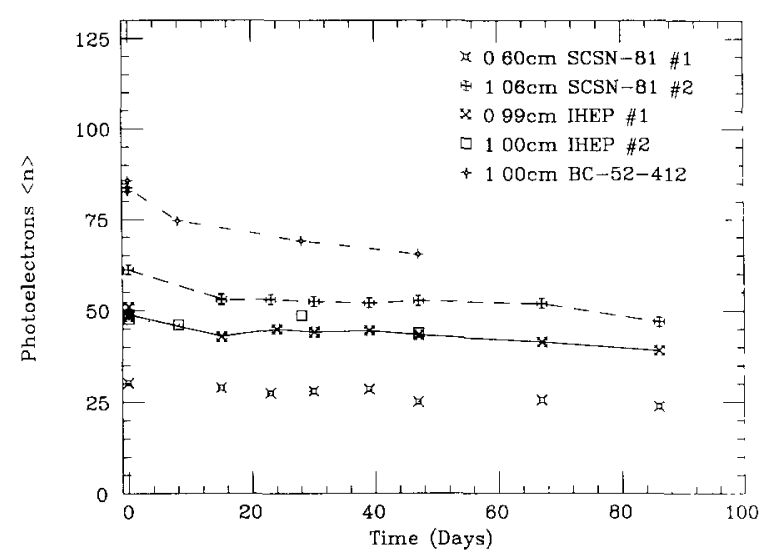

Fig. 16. Response of mechanically stressed polystyrene-based scintillators of various indicated thicknesses as a function of time under stress.

spread from the center toward the ends until about half of the total scintillator area was affected. Small cracks that developed at the bottom of the side edges deepened. After about a month, crazing effects appeared to stabilize. The crazing is not a critical function of stress. A BC-408 piece (labeled No. 2 in Fig. 15) stressed to only $5 \%$ of yield strength crazed as quickly and as severely as the pieces stressed to $10 \%$.

In contrast to the PVT-based scintillators, the PSbased scintillators produced by IHEP and Kuraray showed no visible signs of crazing. An experimental polystyrene scintillator produced by Bicron (BC-52-412) did, however, show some crazing.

To quantify the effect of crazing on counter performance, we periodically measured the photoelectron yield obtaned from cosmic rays traversing the far end of the scintillators. These measurements were performed by briefly removing the scintillator preces from the crazing-test setup and inserting them into a test station consisting of a PMT and cosmic-ray trigger counters. After completion of each measurement, the scintillators were returned to their supports and restressed. Results from these measurements are displayed in Figs. 15 and 16 and show a strong correlation between crazing and loss of light yicld.

When interpreting the results from this crazıng study, it is important to keep in mind that the test pieces were stressed much more severely than in normal applications and that the scintillator most suscept1ble to crazing (BC-408) also gave the highest initial light yield. Nevertheless, crazing is one of the key areas of concern when contemplating the use of scintillators over time intervals measured in decades rather than a few ycars. 


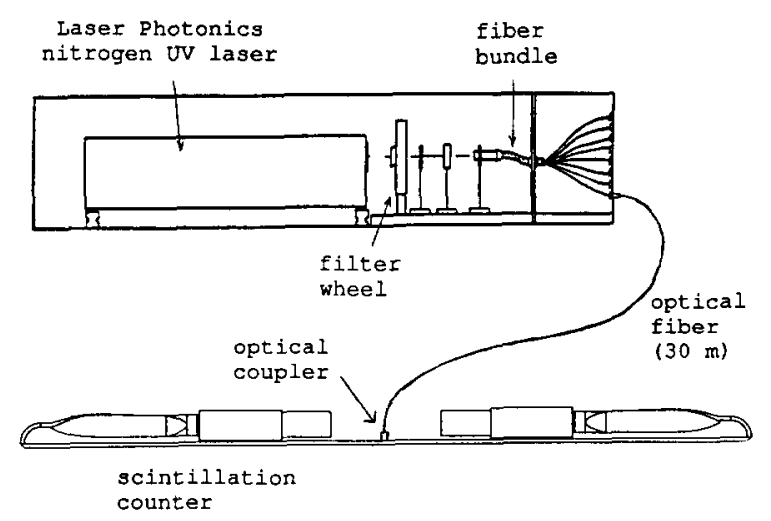

Fig. 17. Layout of proposed laser-based calibration system.

\section{Calibration system}

The SDC muon trigger counters require a calibration system to assure efficient operation and proper synchronization with the data acquisition system. Since the counters are operated in threshold mode, the requirements on monitoring their response are not severe. At the time of SSC termination, the calibration system was still in an early stage of development. Several schemes were under investigation including the use of lasers and LEDs. A system based on UV lasers whose output is distributed simultaneously to many counters is sketched in Fig. 17. One question, unresolved at the time of project termination and primarily a matter of cost, was whether it is possible to measure thresholds sufficiently well (relative to muon signals) without the use of ADCs. In principle, the number of photoelectrons corresponding to a particular threshold can be determined from the response to a fixed calibration signal as a function of threshold setting.

\section{Test facility}

A cosmic-ray facility was constructed and assembled at the SSC Laboratory for testing prototype counters and for validating the performance of all SDC muon trigger counters. The test facility, shown in Fig. 18, consists of three identical planes each of which contains two orthogonal layers of proportional wire chambers (PWCs) and one scintillation trigger counter. The PWC cells, 24 in one layer and 96 in the other, are 2.54 $\mathrm{cm}$ wide. The trigger counter, $66 \mathrm{~cm}$ wide and $250 \mathrm{~cm}$ long, has a design similar to the SDC muon counters and is read out at both ends.

As indicated in Fig. 18, the cosmic-ray facility can measure the photoelectron yield and timing response as a function of position of up to six test counters simultaneously. Operation of the facility requires that

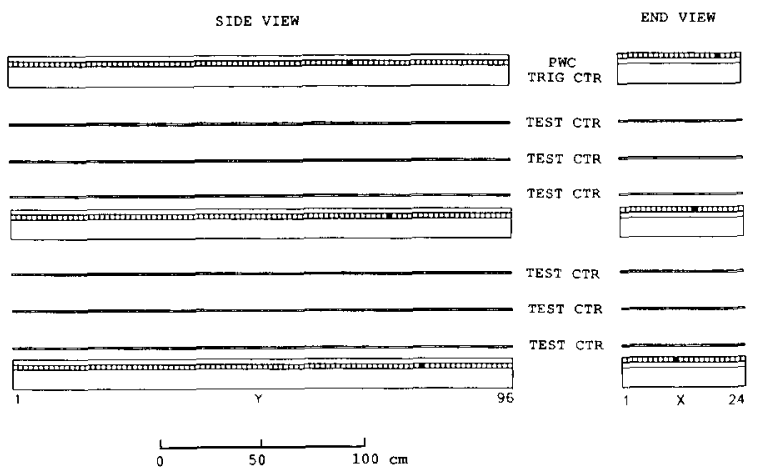

Fig 18. Scale drawing of muon counter test facility assembled at SSCL. A detected cosmic-ray track is indicated by the solid PWC cells.

the three test-stand trigger counters give a coincident signal, yielding a trigger rate of about $50 \mathrm{~Hz}$. In addition, each of the six PWC layers must have one and only one hit in a pattern consistent with a straight track that does not deviate by more than $26^{\circ}$ from the vertical direction. This requirement reduces the overall cosmic-ray rate to about $10 \mathrm{~Hz}$, sufficient to map out the response of muon trigger counters in a few hours.

\section{Backgrounds}

The rate of muons in the barrel muon trigger counters from proton-proton interactions and cosmic rays is estimated to be low, at the $100 \mathrm{~Hz}$ level at the SSC design luminosity of $10^{33} \mathrm{~cm}^{-2} \mathrm{~s}^{-1}$ [13]. The rate produced by low-energy neutrons and gamma rays is expected to be substantially higher than the muon rate and depends critically on the overall shielding configuration of the entire SDC detector.

The neutron detection efficiency of scintillators has been measured [14] and, for a $1.0 \mathrm{~cm}$ thick counter, is reasonably well represented by a function that is essentially zero below neutron energies of $1.0 \mathrm{MeV}$, rises to a maximum of 0.06 at $2 \mathrm{MeV}$, and falls linearly to 0.04 at $10 \mathrm{MeV}$. These values are for perpendicularly incident neutrons and a threshold equivalent to 0.10 of the energy deposited by a muon. Background neutrons have a spectrum with two major peaks, one at thermal energies and the other near $1 \mathrm{MeV}$. The detection rate is dominated by interactions with neutrons having energies of a few $\mathrm{MeV}$. The efficiency for detecting background photons is nearly the same as for neutrons except that the peak of the detection efficiency is shifted down to photon energies just below $1 \mathrm{MeV}$ [15].

Extensive shielding and background studies were carried out by members of the SDC collaboration but 
had not been completed at the time of SSC termination. Preliminary results [16] indicate that the background rates from neutrons and photons are approximately equal and sum to about $10 \mathrm{kHz}$ per barrel muon counter at SSC design luminosity. The effect of the background is to produce spurious triggers at a rate that raises the total muon trigger rate by $5-10 \%$ with no loss of efficiency for muons.

\section{Conclusion}

We designed and tested scintillation trigger counters suitable for use in a large experiment at a highluminosity hadron collider. The counters have good photoelectron yields $(>25)$ and time resolution $(<1$ ns) for muons striking any part of a counter. The use of mean-timing techniques assures coincidence signal arrival times that are independent of where muons traverse the $1.8 \mathrm{~m}$ long counters. Compact light guides that fold back across the scintillator allow good geometric coverage and easy access to all photomultiplier tubes and bases.

\section{Acknowledgements}

This work was supported by the US Department of Energy and SSC Laboratory. We acknowledge R. Ball, B. Goldberg, E. Gero, M. Longo, M. Marcin, H. Schick, A. Simon, and members of the Michigan Physics Instrument Shop for their valuable assistance in carrying out the work reported in this paper. We also thank our SDC colleagues, particularly N. Christensen, C. Daly, K. De, K. Heller, and L. Oesch for their contributions to the SDC muon trigger counter system. At SSCL, A. Fry, C. Mangione, G. Pennycuff, and R. Tanaka gave us outstanding help in setting up the SDC muon counter test facility.

\section{References}

[1] SDC Technical Design Report, SDC-92-201 (1992).

[2] The use of $45^{\circ}$ edges for reflecting scintillation photons was suggested to us by $\mathrm{N}$. Christensen and $\mathrm{K}$. Heller of the University of Minnesota.

[3] HE 17017, manufactured by Hartel Enterprises, 12970 Branford, Pacoima, CA 91331, USA.

[4] Weldon 40, manufactured by IPS Corporation, Gardena, CA 90249 , USA.

[5] BC-630, produced by Bicron Corporation, 12345 Kinsman Road, OH 44065, USA.

[6] BC-634, optical interface disks manufactured by Bicron Corporation.

[7] Optical interface disks were produced at the University of Michigan with General Electric RTV615 according to the general prescription given by L.K. Rangan, E.I. Shibata and J.S. Loos, Nucl. Instr. and Meth. A 276 (1989) 496.

[8] K. Yale et al., SDC-93-597 (1993).

[9] J. Chapman and J. Mann, IEEE Trans. Nucl. Scl. NS-40 (4) (1993) 794

[10] The counters were produced by the IHEP group led by V. Rykalin.

[11] T. Hasegawa et al., Nucl. Instr. and Meth. A 311 (1992) 498.

[12] K. Yale et al., SDC-93-596 (1993).

[13] H. Iwasaki, KEK Preprint 92-161 (1992).

[14] J.E. Hardy, Rev. Sci. Instr. 29 (1958) 705; S.T. Thornton and J.R. Smith, Nucl. Instr. and Meth. 96 (1971) 551;

M. Drosg, Nucl. Instr. and Meth. 105 (1972) 573;

R.A. Cecil et al., Nucl. Instr. and Meth. 161 (1979) 439

[15] Y. Nikolaev and A. Uzunian, SDC-93-512 (1993);

V. Kubarovsky and G. Zholobov, SDC-93-612 (1993).

[16] V. Kubarovsky, SDC-94-613 (1994). 